\title{
SORTING OF CROP RESIDUES AND FOSSIL BONES FROM SOIL BY NIR HYPERSPECTRAL IMAGING
}

\author{
Vincke Damien ${ }^{l}$, Eylenbosch Damien ${ }^{2}$, Fernández Pierna Juan Antonio ${ }^{l}$, Baeten Vincent ${ }^{l}$, Bodson Bernard ${ }^{2}$, Dardenne \\ Pierre $^{l}$ \\ ${ }^{1}$ Walloon Agricultural Research Centre, Department of Valorization of Agricultural Products, Gembloux, Belgium. \\ ${ }^{2}$ Gembloux Agro-Bio Tech - University of Liège, Crop Science Unit, Gembloux, Belgium.
}

\begin{abstract}
The scope of this paper is to present two applications using Near Infrared Hyperspectral Imaging (NIR-HSI) combined with chemometrics to sort constituents of soil and assess their qualitative parameters. In the first application, the feasibility of using NIR-HSI to sort crop residues such as roots and straws in soil has been demonstrated. In the second application the potential of such instrumentation and method to assess the level of collagen preservation in fossil bones has been proved.
\end{abstract}

Index Terms - NIR Hyperspectral Imaging, Soil, Crop residues, bones, PLS-DA

\section{INTRODUCTION}

Soil is a complex matrix containing a wide variety of constituents of interest for disciplines like agronomy or archaeology; it could either be crop residues like roots and straws or bones and ceramics. Current challenges are the detection of these constituents as well as the assessment of their qualitative parameters.

In most cases the traditional methods used for the detection of such constituents requires a manual sorting of the samples or a sieving. In addition, when the qualitative parameters are assessed, it often implies the destruction of the samples or the use of chemical products. For these reasons there is an increasing demand in the development of fast and non-destructive analytical methods able to detect constituents of interest in complex matrix and to assess their qualitative parameters. Near Infrared Hyperspectral Imaging (NIR-HSI) has the potential to meet these needs due to its ability to be applied on-line, allowing the acquisition of large datasets in a short time regardless of sample size and providing information on the distribution of chemical compounds in the sample.

In the last decade, visible and NIR-HSI have become powerful analytical approaches in a number of areas to address environmental, agricultural issues as well as safety evaluation and quality control of agro-food products, e.g.: to detect meat and bone meal in animal feed to help prevent Bovine Spongiform Encephalopathy (BSE) [1] or other contaminants like ergot bodies in cereals [2].
In the field of soil analyses, hyperspectral imaging has been applied to analyse soil organic matter and phosphorus [3-7], to determine maize and pasture root density [8-9], or to classify archaeological soils [10].

\section{INSTRUMENTATION}

In these studies, a NIR hyperspectral line scan (also called push-broom imaging or NIR-HSI) system combined with a conveyor belt (BurgerMetrics SIA, Riga, Latvia) was used (see Figure 1). The instrument is a SWIR XEVA CL 2.5320 TE4 camera (Specim Ltd, Oulu, Finland); using an ImSpector N25E spectrograph that includes a cooled, temperature-stabilized Mercury-Cadmium-Telluride (MCT) detector (Xenics nv, Leuven, Belgium). The system projects a beam of light onto a two-dimensional Focal Plane Array (FPA) and each image consists of 320-pixel lines acquired in the range $1100-2400 \mathrm{~nm}$ with an interval of $6,3 \mathrm{~nm} .32$ scans per image have been averaged and each pixel provides a reflectance spectrum of a point of the scene [11]. Acquisition is done using HyperPro software (BurgerMetrics SIA, Riga, Latvia).

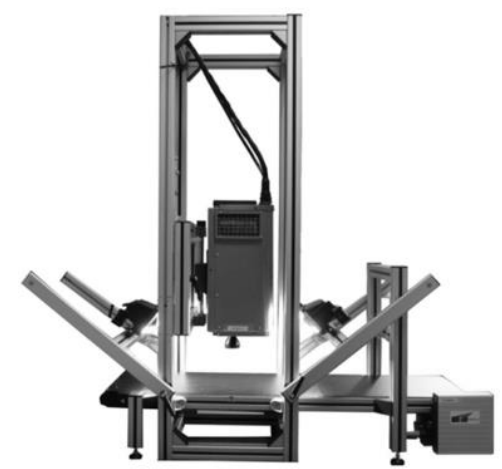

Figure 1: NIR Hyperspectral Imaging system.

Prior to analysis, the spectral NIR-HSI system is calibrated with a dark image (by shutting off the lens entrance) and a white image (background) collected from a standard white reference board (empty teflon plate). The spectra are then automatically corrected. This procedure is performed to compensate for offset due to the light source temperature drift, and the lack of spatial lighting uniformity. 


\section{DETECTION OF CROP RESIDUES IN SOIL SAMPLES}

Current challenges in agricultural research focus on the effect of tillage and the quantification of roots and straw residues in soil, which are key constituents for the monitoring of the development of the root systems and the decomposition of crop residues. In this context, a tentative study has been performed using NIR-HIS and chemometric tools to characterize soil sediments and to detect and quantify roots and straws.

Chemometric methods like Partial Least Squares Discriminant Analysis (PLS-DA) allow the extraction of the maximum amount of information from the raw data [12]. In the present study a hierarchical classification tree based on four classes (background, soil, roots and straws) has been constructed [13]. The classification has been allowed by "successive exclusions" using binary PLS-DA models constructed on a spectral databank of each class. The first step of the tree classification used a model to remove spectra related to background (conveyor belt and sieve) (Figure 2); the second step separated soil from straws and roots and the last step discriminated 'straws' and 'roots' spectra. These models have been created with a set of 1000 spectra for each class selected using the duplex method [14] and a set containing 500 independent spectra by class has been used to validate them. All the spectra were pre-processed by applying an autoscale correction. After validation, the models have been applied to new images of complex mixtures of soil constituents (Figure 3).

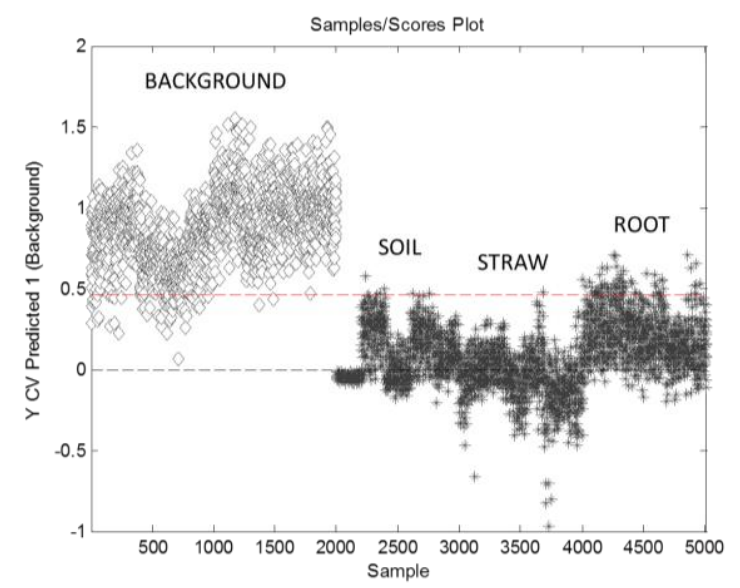

Figure 2: Example of PLS-DA model to discriminate background (conveyor belt and sieve) from the rest (soil, straws and roots).

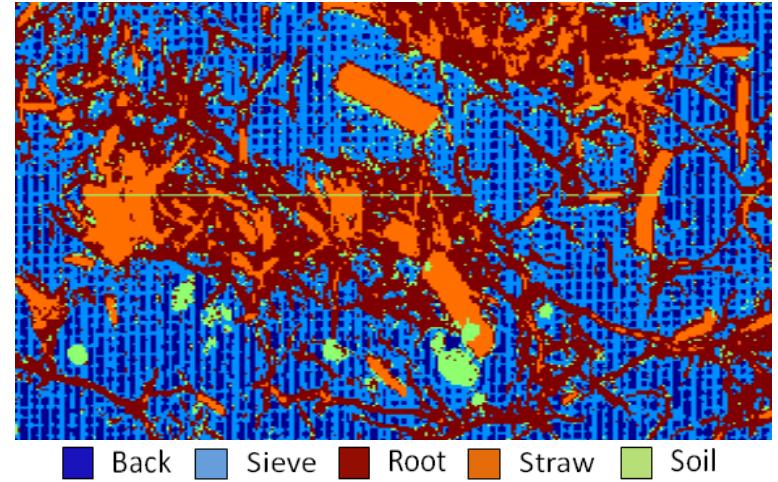

Figure 3: prediction by the PLS-DA models of a complex image containing all constituents used in models construction.

The preliminary results obtained demonstrated the feasibility of a detection method, based on the spectra, for the presence of different constituents (roots and straws) in a soil sample. This is the first step for a possible quantification of each constituent. To achieve such quantification, further research has to be done by linking the prediction of individual pixels on the NIR images with the corresponding weight of the constituent in the sample. This study will be crucial for the monitoring of root development and organic matter decomposition in soil.

\section{EVALUATION OF COLLAGEN PRESERVATION IN FOSSIL BONES}

In archaeology many analyses require collagen like, the Zooarchaeology by Mass Spectrometry (ZooMS) technique for taxonomic identification, the radiocarbon (AMS) dating and stable isotopic analyses to reconstruct past human and animal diets.

For these analyses, the challenge is to obtain bone samples with sufficient collagen content to get positive results. To date, there has been no protocol or analytic method capable of rapidly and non-destructively screen bones to detect and quantify collagen. In this context, the advantages of NIR-HSI represent a high potential for the development of a new analytical method [15].

The success or failure of $\mathrm{C} 14$ dating of bones and their geological context were used as criteria to define two classes of bones with sufficient collagen (successful dating, non-alluvial layers) and with little or no collagen (failed dating, alluvial layers i.e. removal of collagen by water leaching).

A sample set of 16 bones ( 8 for each aforementioned class) was used to create a spectral library of 400 spectra for each class. The spectral libraries have been randomly collected by achieving a representative subsampling of 50 spectra for each bone on both sides of the bone (25 spectra per side). The mean spectra of each class are illustrated in 
Figure 4. This figure also presents the mean spectrum of a bone from a medieval cow (standard sample) for which chemical analyses indicated a high level of collagen. The comparison of this reference spectrum with the mean spectrum of bone with collagen $(\mathrm{COL}+)$ shows some similarities in the range $2000-2300 \mathrm{~nm}$ (vertical lines in figure 4). In addition spectral differences are observed between bones with and without collagen (respectively COL+ and COL- in figure 4).

In a first step, a PLS-DA model has been built to discriminate the bones on the basis of their collagen content. The sample set of 16 bones previously mentioned was used to calibrate the model and 4 new samples from another set ( 2 of each class) were predicted to validate the model.

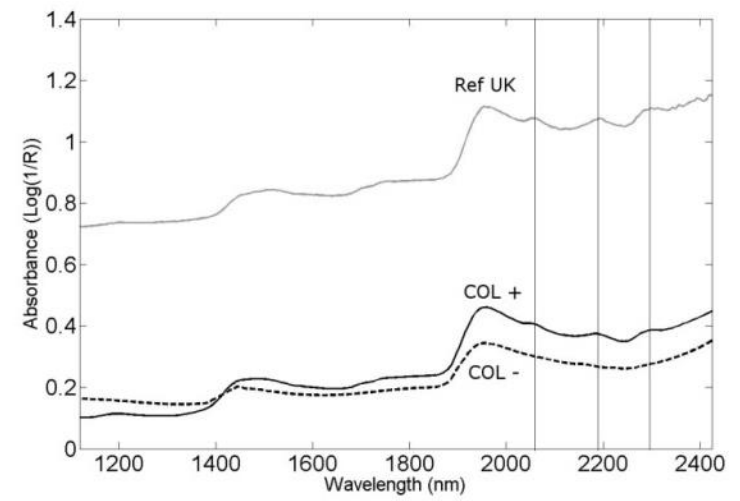

Figure 4: mean spectra of samples with sufficient (COL+), insufficient collagen (COL-) and reference bone (ref UK).

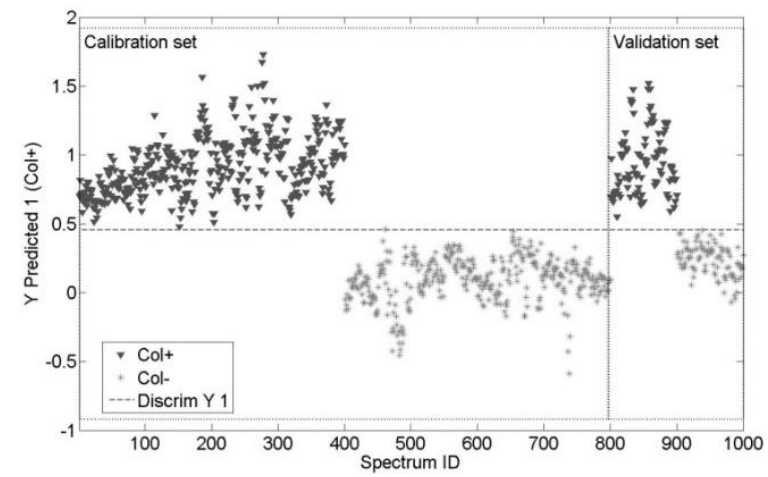

Figure 5: calibration and validation of the PLS-DA model.

The results presented in Figure 5 show that all of the new samples were correctly sorted by the model. This demonstrates the ability of the model to detect the presence or absence of collagen in the bones.

In a second step, the model has been applied to two independent sets of samples from two different strata in order to compare the collagen preservation status of the bones within each stratum.
Figure 6 and Figure 7 present the mean prediction scores of each sample plotted with their respective standard deviation. The dashed line drawn on these figures represents the threshold used by the model to sort a sample as containing collagen. A sample sorted above this threshold indicates that collagen have been detected within it.

In Figure 6 almost all the samples have been sorted below the dashed line which means that no or only small amounts of collagen have been detected. These results are consistent with the geological information recorded for this stratum, which indicated that it has been subjected to recurrent water flooding and therefore could lead to the removal of the collagen from the bones.

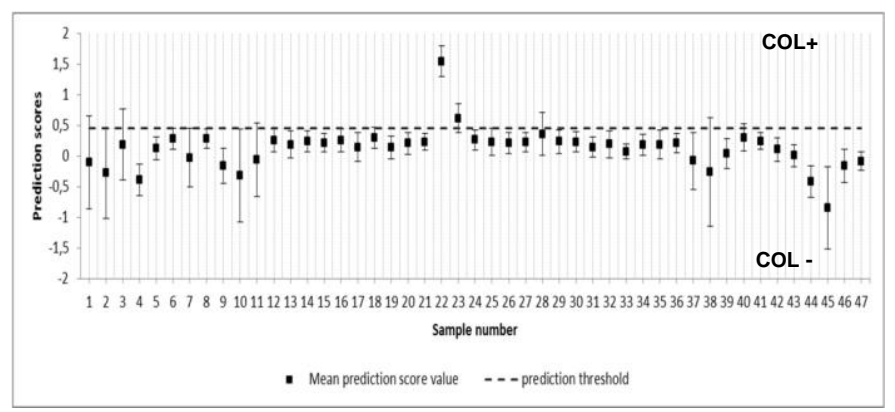

Figure 6: PLS-DA predictions for samples from an alluvial layer

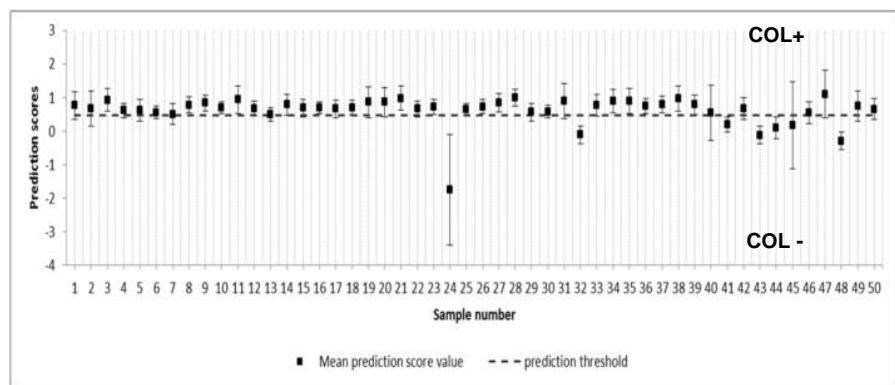

Figure 7: PLS-DA predictions for samples from a non-alluvial layer

In Figure 7 almost all the samples have been sorted above the dashed line indicating that collagen has been detected. Again this result is supported by the geological information since this stratum has not been subjected to recurrent water flooding and it could be assumed that the collagen of these bones is preserved.

\section{CONCLUSION}

In the present work, two cases studies have been presented. The first one concerns a feasibility study where NIR-HSI combined with chemometrics has been proved to be a good alternative as classical methods for sorting the different constituents of soils like crop residues (roots and straws). In the second case study, the potential of the technique to assess the level of collagen preservation in 
fossil bones has been proved. In both cases, only qualitative results have been obtained. The future challenge will be the possible quantification of the constituents. In the case of the crop residues it will be to quantify the respective weights of each constituent (roots and straws) and in the case of the collagen in fossil bones, it will be the assessment of the amount of collagen present.

\section{ACKNOWLEDGEMENTS}

The ArcheoNIR project is funded by the Fonds de la Recherche Scientifique (FNRS), Fonds de la recherche Fondamentale Collective (FRFC), project number F.FRFC 2.4621.12. The Trou Al'Wesse project, directed by Rebecca Miller and Marcel Otte (University of Liège, Service of Prehistory, Belgium), is supported by annual subsidies $(02 / 16341$ to $13 / 19227)$ granted by the Service public de Wallonie (SPW). We are also grateful to Ouissam Abbas (CRA-W) and the technical staff of all of the teams.

\section{REFERENCES}

[1] J.A. Fernández Pierna, P. Dardenne and V. Baeten, "Inhouse validation of a near infrared hyperspectral imaging method for detecting preprocessed animal proteins in compound feed", ,Journal of Near Infrared Spectroscopy, IM Publications, pp. 121-133, May 2010.

[2] P. Vermeulen, J.A. Fernández Pierna, H.P. van Egmond, J. Zegers, P. Dardenne and V. Baeten "Validation and transferability study of a method based on near-infrared hyperspectral imaging for the detection and quantification of ergot bodies in cereals," Analytical and Bioanaytical Chemistry, Springer, pp. 7765-7772, 2013.

[3] M. Steffens and H. Buddenbaum, "Laboratory imaging spectroscopy of a stagnic Luvisol profile - High resolution soil characterisation, classification and mapping of elemental concentrations", Geoderma, Elsevier, pp. 122-132 December 2013.

[4] M.R. Maleki, A.M. Mouazen, B. De Ketelaere, H. Ramon and J. De Baerdemaeker, "On-the-go variable-rate phosphorus fertilisation based on a visible and near-infrared soil sensor", Biosystems Engineering, Elsevier, pp. 35-46, 2008

[5] A.M. Mouazen, M.R. Maleki, J. De Baerdemaeker and H. Ramon, "On-line measurement of some selected soil properties using a VIS-NIR sensor", Soil and Tillage Research, Elsevier, pp. 13-27, 2007.

[6] R.A.Viscarra Rossel, D.J.J. Walvoort, A.B. McBratney, L.J. Janik and J.O. Skjemstad, "Visible, near infrared, mid infrared or combined diffuse reflectance spectroscopy for simultaneous assessment of various soil properties", Geoderma, Elsevier, pp. 59-75, 2006.

[7] R.A. Viscarra Rossel, Y. Fouad and C. Walter, "Using a digital camera to measure soil organic carbon and iron contents", Biosystems Engineering, Elsevier, pp. 149-159, 2008.

[8] B.H. Kusumo, M.J. Hedley, C.B. Hedley and M.P. Tuohy "Measuring carbon dynamics in field soils using soil spectral reflectance: prediction of maize root density, soil organic carbon and nitrogen content", Plant and Soil, Spinger, pp. 233-245, 2011.

[9] B.H. Kusumo, M.J. Hedley, C.B. Hedley, G.C. Arnold and M.P. Tuohy "Predicting pasture root density from soil spectral reflectance: field measurement," European Journal of Soil Science, Wiley, pp. 1-13, 2010.

[10] J. Linderholm \& P. Geladi, "Classification of archaeological soil and sediment samples using near infrared techniques." NIR news, IM Publications, pp. 6-9, 2012.

[11] P. Vermeulen, J.A. Fernández Pierna J.A., H.P. van Egmond, P. Dardenne and V. Baeten, 2012 "Online detection and quantification of ergot bodies in cereals using near infrared hyperspectral imaging", Food Additives \& Contaminants: Part A: Chemistry, Analysis, Control, Exposure \& amp; Risk Assessment, pp. 232-240.

[12] J.A. Fernández Pierna, V. Baeten, J. Dubois, J. Burger, E.N. Lewis and P. Dardenne, Comprehensive Chemometrics, Elsevier, Oxford, 2009.

[13] J.A. Fernández Pierna, V. Baeten and P. Dardenne "Screening of compound feeds using NIR hyperspectral data", Chemometrics and intelligent laboratory systems, Elsevier, pp. 114-118, 2006.

[14] R.D. Snee, "Validation of Regression Models: Methods and Examples", Technometrics, American Statistical Association, pp. 415-428, 1977.

[15] D. Vincke, R. Miller, E. Stassart, M. Otte, P. Dardenne, M. Collins, K. Wilkinson, J. Stewart, V. Baeten and J.A. Fernández Pierna, "Analysis of collagen preservation in bones recovered in archaeological contexts using NIR hyperspectral imaging", Talanta, Elsevier, pp. 181-188, July 2014. 